\title{
EDITORIAL
}

\section{EL MOMENTO ES AHORA, NURSING NOW}

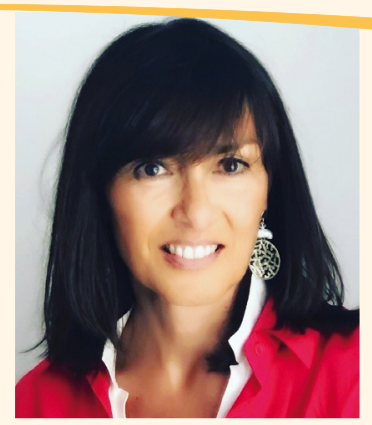

ZULEMA GANCEDO GONZÁLEZ

Enfermera.

Servicio Cántabro de Salud. Nursing Now Cantabria.
El análisis de la situación mundial respecto al compromiso con los Objetivos de Desarrollo Sostenible (ODS) y su alcance, la escasez de recursos, de profesionales y, en concreto, la escasez de enfermeras, sus condiciones laborales y de desarrollo profesional provocan la necesidad de impulsar un movimiento a nivel mundial como lo es la campaña Nursing Now. Un gran reto para las enfermeras del mundo y también para los países comprometidos con la salud, el bienestar y la lucha contra las enfermedades. El arranque de la campaña se inicia en enero de 2018 desde el Reino Unido a través de un acto seguido desde diferentes países, y que sirvió como preludio para la suma en cadena de otros, como es el caso de España. Actualmente, ya ha alcanzado la difusión internacional que se había previsto. Más de 120 países adheridos, 587 grupos involucrados y 689 empleadores, que, a fecha actual, han aceptado formar a 26236 enfermeras y matronas a través del programa Nightingale Challenge. Un reto de formación en liderazgo en el contexto institucional, que pretende formar a enfermeras jóvenes con el fin de prepararlas para el futuro, desarrollar su potencial y afrontar los desafíos en salud del siglo xxı. Nunca antes se había dado un movimiento internacional de tanto calado e interés para los distintos países y las comunidades ${ }^{1}$.

En el contexto mundial y analizando los factores y causas para enfermedad y muerte, observamos como, en los países desarrollados, las causas de mortalidad no han variado en los últimos años: cáncer, enfermedades cardiovasculares, respiratorias, diabetes... alcanzaron el $73 \%$ de la mortalidad mundial respecto a enfermedades no transmisibles en $2017^{2,3}$. El estilo de vida y el entorno marcan las causas del desarrollo de estas enfermedades atribuibles a factores como el tabaco, el consumo de alcohol, la obesidad y la hipertensión arterial. En el caso de la salud mental, los trastornos depresivos constituyen la mayor causa de discapacidad y enfermedad en el mundo y estos trastornos no diferencian entre niveles sociales o económicos. Epidemias, otras enfermedades endémicas que han ido llegando de otros países con la globalización y los movimientos migratorios, y la propia demografía de estos últimos a causa de conflictos, guerras, hambrunas o ausencia de derechos generan en los países receptores verdaderas crisis sobre la cobertura y asis- 
tencia sanitaria en una población sin recursos, con necesidades y notables diferencias culturales y sociales. Un estado de vulnerabilidad global que pone en jaque a los sistemas sanitarios y a los gobiernos de los países ${ }^{4}$.

En estos momentos, la campaña se antoja como una auténtica encrucijada ante perspectivas diferentes de respuesta y dos factores esenciales: los contextos sociopolíticos, económicos y culturales de los diversos países, y el grado de implicación de los distintos actores en dicha campaña, impulsores directos, políticos y gestores y, sin duda, los propios profesionales. En este sentido, debemos ser conscientes de que las realidades en los diferentes países del mundo son bien distintas. Las diferencias entre naciones, tanto en la riqueza como en su distribución, el diseño e inversión en planes de salud, salud pública en general, y también el papel o funciones de sus profesionales marcan distancias respecto a la cobertura sanitaria y los programas de salud, el acceso y la atención, la diversidad y el nivel de desigualdad. Por lo tanto, cada uno de ellos, y con el marco de la campaña, principios, valores, programas que trabajar y objetivos que la sustentan, deberán tener en cuenta cuestiones que se adapten a la mejora de su realidad.

Un apunte certero y determinante en salud global y acerca de las enfermeras es que, independientemente de las funciones que desempeñen en su país, el común denominador son los problemas o factores que impiden un desarrollo y participación plena. Estos factores han sido identificados y estudiados detallándose en el informe «Triple Impacto», un informe concluyente elaborado por un grupo de trabajo sobre salud global del Parlamento Británico y coordinado por Lord Crisp, experto en salud global y desarrollo internacional, y también actual copresidente de Nursing Now ${ }^{1,5}$. Este consenso sobre la problemática común de la profesión a nivel mundial tiene consecuencias nada despreciables en salud. Justamente el informe «Triple Impacto» concluye, además, que es necesario empoderar a las enfermeras dándoles participación, apoyándolas, invirtiendo en su formación y capacitación, en su desarrollo y sus entornos laborales. Es necesario permitirles desarrollar el ejercicio del liderazgo a todos los niveles si se quieren alcanzar objetivos en salud, economía y equidad de género, contribuciones necesarias para el compromiso con los ODS, con la Agenda 2030 y necesariamente con el progreso social y económico, y para una mayor libertad y paz mundial6.

En términos generales, es bien sabido que los problemas de salud de la población, y los psicosociales también, vienen determinados por las distintas políticas establecidas en cada país. Políticas económicas, sociales y sanitarias determinan o limitan los recursos, afectando y condicionando el acceso a los servicios, generando desigualdades y, en ese contexto, también el grado de profesionalización de las enfermeras y la escasez de estas afecta a la salud de la población de los países. Esta escasez de profesionales junto con la precariedad laboral, el abandono de la profesión, la migración, y el escaso apoyo e inversión en el desarrollo y mejora profesional determinan una problemática para abordar ${ }^{5}$.

A estos mismos problemas, se suman otras dificultades también identificadas como obstáculos que impiden alcanzar sus metas: limitaciones en la capacidad para resolver problemas de salud, limitaciones en el desarrollo profesional y laboral, en la propia autonomía y la baja o nula participación en análisis y estudios del medio, de situaciones que ellas conocen y manejan y, por ende, tampoco en la planificación de políticas al respecto. La escasa «voz» en el espacio público con derecho a decir y ser escuchado, el escaso protagonismo y presencia, las limitaciones impuestas por modelos «agotados», que no aceptan reformas profundas y acordes con la realidad, y la constante y displicente mirada de otros colectivos son también obstáculos necesarios para abordar. Podemos concluir que su voz no es escuchada, aun cuando su mirada es global, analítica, fundamental y necesaria para la atención en el contexto actual de la salud y lo psicosocial, ambos determinantes bidireccionales ${ }^{1,2,5-7}$. 
Se considera que las enfermeras, por su formación y visión humanista, son garantes y generadoras de salud en las comunidades ${ }^{6}$ y que, por lo tanto, contribuyen a mejorar las economías, a garantizar el acceso a la salud, a la sostenibilidad de los sistemas y a la equidad de género ${ }^{5}$. Ellas son garantía de cobertura sanitaria universal por esencia y responsabilidad ética profesional a través del derecho a la salud, y ese ha sido el posicionamiento constante desde el Consejo Internacional de Enfermeras o CIE (ICN, por las siglas en inglés de International Council of Nurses), a través de sus políticas, declaraciones y posicionamientos, cuyos lemas engloban siempre «salud para todos» ${ }^{6,7}$.

$\mathrm{Si}$, bajo este prisma, las estrategias y la planificación en salud no conllevan implícitas las consideraciones enfermeras, difícilmente pueden contemplar la visión, el conocimiento específico y la experiencia de los profesionales que más cerca están del individuo, las familias y la comunidad. Por lo tanto y en definitiva, la infravaloración e infrautilización profesional sistemática tiene un alto impacto en la salud y en la calidad de vida de las personas y las comunidades.

Se necesitan enfermeras para generar equilibrio entre la oportunidad de acción y las de facto, para facilitar el acceso a los servicios sanitarios, para aumentar las intervenciones en promoción y educación para la salud, para cubrir las necesidades actuales a través de la práctica avanzada, para generar evidencia y trasladarla a la práctica clínica, para compartirla y que sirva para la toma de decisiones en salud. La contribución de las enfermeras facilita la consecución de los objetivos y el logro de los distintos retos en salud global.

Los contextos y factores que determinan las diferencias entre países pueden ser abordables en mayor o menor medida, por ello, se requiere voluntad y compromiso político, tal y como la Organización Mundial de la Salud promulga, instando a los gobiernos a mejorar las condiciones laborales y de desarrollo y facilitando mecanismos para restar los obstáculos que les impiden progresar. Dar voz y participación en igualdad de oportunidades y condiciones, y garantizar la presencia en todos los foros, debates y toma de decisiones en políticas sanitarias y como profesionales de la salud'.

Los objetivos marcados por la campaña son tan globales, y a la vez tan ajustados a la problemática compartida de las enfermeras, que permiten que cada país, región o comunidad defina las acciones necesarias para trabajar en cada uno de los programas y llegar a la diversidad de la población. De hecho, parece que las actuaciones que se han derivado ya de la campaña han tenido más repercusión en países donde las enfermeras no tienen un alto nivel de profesionalización, ni políticas de salud tan desarrolladas en cuanto a cobertura y recursos.

En nuestro entorno y desde los diferentes grupos que se han ido formando, se ha trabajado, sobre todo, en la difusión de la campaña, en el análisis de la situación desde la perspectiva profesional, y la de necesidades en salud de la población. Cada grupo ha concluido una serie de propuestas que contemplan: mejoras profesionales sobre la práctica clínica, sobre los entornos laborales, sobre regulaciones y normativas, sobre la revisión de procesos y estrategias para mejorar la atención y la salud de la población, con una justa participación y con mayor protagonismo en estos, y también sobre cómo recuperar la inversión perdida en dichas mejoras. Asimismo, se han formulado propuestas imprescindibles sobre cómo hacer visible nuestro trabajo, el impacto de nuestras actuaciones, el fomento y transmisión del liderazgo, la práctica avanzada y especializada. Todas las propuestas se plantean con evidencia y estudios próximos y validados. Se han trabajado canales de comunicación alojados en medios como revistas científicas, redes sociales con canales propios, foros, debates y participaciones en congresos y reuniones para poner voz y llegar a todos los profesionales y responsables. Se han mantenido contactos con políticos y responsables, aunque, desde mi perspectiva y juicio personal, el gran paso está por dar, esto es, la alianza firme con los ciudadanos, la sociedad y la población en general. 
Tan sencillo y tan difícil, una apuesta clave para que las enfermeras puedan garantizar con su contribución un futuro mejor para todos, pendientes de sacar partido a nuestro máximo potencial, nuestro mejor talento y a un ejercicio pleno de nuestro liderazgo.

\section{BIBLIOGRAFÍA}

1. Nursing now [Internet]. Londres: Campaña Nursing Now; 2019. Disponible en: http://www.nursingnow.org/

2. The Lancet. GBD 2017: a fragile world. Lancet. 2018;392(10159):1683.

3. GBD 2017 Disease and Injury Incidence and Prevalence Collaborators. Global, regional, and national incidence, prevalence, and years lived with disability for 354 diseases and injuries for 195 countries and territories, 1990-2017: a systematic analysis for the Global Burden of Disease Study 2017. Lancet. 2018;392(10159):1789-858.

4. Stewart D, Burton E, White J, Salmon M, McClelland A. Health for all nursing, global health and universal health coverage. International Nurses Day of 2019. Resources and evidences. International Council of Nurses. Ginebra: ICN-International Council of Nurses; 2019. Disponible en: https://2019.icnvoicetolead.com/wp-content/uploads/2017/04/ ICN_Design_EN.pdf

5. All-Party Parliamentary Group on Global Health. Triple Impact. How developing nursing will improve health, promote gender equality and support economic growth. Londres: All-Party Parliamentary Group on Global Health (APPG); 2016. Disponible en: https://www.who.int/hrh/com-heeg/digital-APPG_triple-impact.pdf

6. World Health Organization. Health workforce. Triple Impact - how developing nursing will improve health, promote gender equality and support economic growth [Internet]. Disponible en: https://www.who.int/hrh/com-heeg/triple-impactappg/en/

7. Stilwell B. \#Nursing Now. Creat Nurs. 2019;25(1):6-9. 